\title{
TTR
}

Traduction, terminologie, re?daction

\section{Discordance dans la concordance : ressacs de la traduction}

\section{Patrick J. Mahony}

Volume 11, numéro 2, 2e semestre 1998

Psychanalyse et traduction : voies de traverse

Psychoanalysis and Translation: Passages Between and Beyond

URI : https://id.erudit.org/iderudit/037333ar

DOI : https://doi.org/10.7202/037333ar

Aller au sommaire du numéro

\section{Éditeur(s)}

Association canadienne de traductologie

ISSN

0835-8443 (imprimé)

1708-2188 (numérique)

Découvrir la revue

Citer cet article

Mahony, P. J. (1998). Discordance dans la concordance : ressacs de la traduction. TTR, 11(2), 39-53. https://doi.org/10.7202/037333ar

\section{Résumé de l'article}

Discordance dans la concordance : ressacs de la traduction - L'auteur décrit d'abord le dessein et les propriétés de la Konkordanz, monumental ouvrage de référence paru en 1996. Véritable événement éditorial dans le champ psychanalytique, cet ouvrage donne accès aux termes clés des Gesammelte Werke de Freud. L'auteur compare ensuite cette Konkordanz allemande et la Concordance anglaise, et relève des discordances révélatrices sur le plan lexicographique. Cette comparaison confirme la remarquable cohérence de la conceptualité freudienne, d'une part, et la nécessité d'entreprendre une «psychanalyse de la traduction de Strachey » d'autre part, car les processus de l'inconscient et du préconscient ne passent pas de la même manière d'une langue à l'autre. L'anglais doit-il rester la lingua franca de la psychanalyse?

Tous droits réservés ( $\odot$ TTR: traduction, terminologie, rédaction — Les auteurs, Ce document est protégé par la loi sur le droit d'auteur. L’utilisation des 1998

services d'Érudit (y compris la reproduction) est assujettie à sa politique d'utilisation que vous pouvez consulter en ligne.

https://apropos.erudit.org/fr/usagers/politique-dutilisation/ 


\title{
Discordance dans la concordance : ressacs de la traduction
}

\author{
Patrick J. Mahony
}

Traduction de Patrick Poirier

Seule une métaphore empruntée à l'astronomie peut rendre compte de l'événement. Dans le champ des études freudiennes, une étoile vient de naître : la Konkordanz ${ }^{1}$. Grâce aux avancées de l'informatique et au dévouement de nombreux chercheurs, nous avons en effet maintenant accès à un index alphabétique exhaustif de tous les mots clés des Gesammelte Werke, l'édition allemande de l'cuuve complète de Freud. C'est dire à quel point la Konkordanz s'avérera un outil indispensable pour les analystes et les exégètes qui souhaiteraient retracer avec plus de précision l'évolution des concepts, de l'imagerie et des associations métaphoriques de la pensée freudienne (leur développement comme leur abandon), de même, et tout praticulièrement, que les figures associatives sur lesquelles reposent les théries de Freud. Il est donc clair que toutes les sociétés psychanalytiques dignes de ce nom, de même que toute bibliothèque de qualité dédiée à l'histoire de la pensée, devront posséder cet indispensable outil. Et si son prix n'est pas négligeable (il toume autour de 1390 \$ à l'heure actuelle), l'usage informé de la Konkordanz offre, en retour, d'inestimables avantages.

' Cet article, paru en anglais dans la revue International Journal of PsychoAnalysis (1996, 77, pp. 407-412) soulignait la parution alors récente de la somme intitulée Konkordanz zu den Gesammelten Werken von Sigmund Freud (Edited by Sarnuel Guttman et al. Six volumes. Waterloo, Canada, North Waterloo Academic Press, 1996, 6800 p.). 
Un premier avantage pratique de la Konkordanz tient à la possibilité de pouvoir retracer rapidement un mot en remontant à sa source parfois oubliée. Un autre avantage, plus intéressant encore pour la compréhension de l'œuvre, consiste dans le fait que l'ouvrage s'avère être une base sûre nous permettant d'étudier la terminologie et les concepts freudiens à la fois dans leur sens historique et dans leur devenir. Ainsi, les cliniciens et les chercheurs des différentes disciplines influencées par Freud peuvent maintenant avoir une meilleure compréhension de sa juste contribution à leur domaine. De plus, ils ont désormais en main un puissant instrument leur permettant non seulement de nettoyer le bourbier d'erreurs interprétatives qui, à ce jour, foisonnent encore dans les cercles académiques et psychanalytiques, mais encore de faire d'abondantes découvertes quant aux processus psychiques - conscients et inconscients - de Freud. Nous ne devrions toutefois pas nous engager dans une telle entreprise en vue d'une quelconque exégèse infinie - et comme telle banalisante - de l'œuvre, ni entretenir l'illusion selon laquelle, Freud ayant écrit une ouvre essentiellement scientifique, les éléments soi-disant esthétiques de ce discours seraient marginaux et insignifiants. Faut-il rappeler que, écrit sous la pression d'une urgence intérieure et ne cherchant pas nécessairement à véhiculer un message clair d'un texte à l'autre, le discours de Freud porte constamment en lui-même un autre sens (Mahony, 1987a, b; 1989; 1995)?

Quelques mots s'imposent en ce qui concerne le dessein et le format de la Konkordanz. L'ouvrage, qui compte six volumes, répertorie dans leur contexte plus d'un million de mots et de phrases apparaissant dans les Gesammelte Werke. En ce sens, on comprendra qu'un des intérêts premiers de la Konkordanz consiste dans sa double organisation alphabétique : disposé selon cet ordre, chacun des mots-clés est cité dans son contexte et chaque variante a sa propre entrée. Les contextes qui accompagnent ces mots-clés sont également alphabétisés. De plus, chaque ligne de contexte renvoie le lecteur au volume et à la page correspondante dans les Gesammelte Werke, suivi de l'abrégé du titre de sa source et sa date de parution.

On comprendra mieux à partir du double exemple suivant :

ziehen, daß ein natürlicher Instinkt zrm Inzest treibt, und $9150 \mathrm{~b}$ Totem Tabu 1913 
daß der Inzest diesem Instinkt zum Trotz kein seltenes 9 148a Totem Tabu 1913

Le lecteur notera que "Instinkt " apparaît dans Totem et Tabou aux pages 148 et 150 du neuvième volume des Gesammelte Werke. La raison pour laquelle l'exemple de la page 150 est répertorié avant celui de la page 148 tient au contexte qui suit "Instinkt " : alphabétiquement, " zum Inzest " précède * zum Trotz $n$. Une telle stratégie éditoriale s'avère particulièrement utile lorsqu'on se souvient d'un mot très fréquent et des termes qui le suivent, mais dont on aurait oublié la source.

Dans la Concordance anglaise, chaque page est divisée en quatre parties $(a, b, c, d)$, de sorte que si, par exemple, le mot * instinct » est indexé " $13122 \mathrm{c}$ ", c'est qu'il apparaît dans le troisième quart de la page 122 du treizième volume de la Standard Edition. Comme la Konkordanz allemande ne divise la page qu'en deux parties $(a, b)$, il faut mettre un peu plus de temps pour retrouver le mot recherché dans les Gesammelte Werke. Malgré ce léger inconvénient, les lecteurs des traductions comparées pourront néanmoins tirer grand usage du répertoire complet de conversion des paginations que l'on retrouve dans la Concordance anglaise, liste qui fournit les pages et les volumes correspondants des deux ouvrages, anglais et allemand.

Il caut toutefois mettre le lecteur en garde. Traduction, trahison; concordance, discordance. Les chercheurs vont rapidement prendre conscience d'un fait pour le moins ironique, mais extrêmement révélateur : quelles que soient les similitudes entre les * concordances * anglaise et allemande, il reste, à un autre niveau - plus important encore - , des différences et des discordances ayant trait aux éditions de référence des deux langues. En dépit de son statut de monument, qu'elle doit en partie à un splendide travail éditorial et au fait qu'on y retrouve de nombreuses variantes et corrections apportées par Freud a ses textes, la English Standard Edition de l'œuvre de Freud, s'il faut parler en termes de critères bibliographiques, est tout sauf une édition standard. La " Standard "Edition, intitulé le plus trompeur de notre domaine, conférait en effet un certain confort, une pseudo-certitude, voire un attrait narcissique aux psychanalystes. Néanmoins, parce qu'elle contenait trenteneuf textes de plus que les Gesammelte Werke, la Standard Edition de Strachey faisait preuve d'une certaine supériorité intrinsèque et, de fait, 
l'ouvrage a connu et profité d'un statut canonique qui, en 1980, allait être renforcé par la publication de la English Concordance. En 1987 par contre - et je fais référence aux Gesammelte Werke plutôt qu'à toute autre édition allemande -, un renversement se faisait jour dans le déséquilibre éditorial. En plus de ses améliorations bibliographiques impressionnantes, le dix-huitième volume des Gesammelte Werke rassemblait en fait un certain nombre de nouvelles publications que l'on ne retrouve pas dans la Standard Edition. De sorte que, grâce aux nouveaux ajouts et aux améliorations des Gesammelte Werke, la Konkordanz a fini par miner davantage le statut canonique de la Standard Edition qui, après tout, et dans tous les sens du mot, n'est qu'un simple dérivé lexical.

Parmi les particularités qui biaisent toute comparaison quantitative entre les inventaires des deux ouvrages, je pourrais souligner que seule la Concordance anglaise répertorie le contenu des lettres que Freud écrivit à Fliess (quelque cent pages de cette correspondance sont publiées dans le premier volume de la Standard Edition). De plus, si on le compare avec le demier volume des Gesammelte Werke, qui contient la totalité des notes de Freud sur l'Homme aux rats, la Standard Edition n'en retient que le tiers - ce que j'ai déjà longuement commenté ailleurs (1986). En plus d'utiliser des sources textuelles divergentes, Strachey, d'après mes estimations sommaires, a par inadvertance oublié de traduire près de 3000 mots du texte allemand. En d'autres occasions encore, il a inutilement rendu sa traduction ou trop verbeuse ou trop abrégée (incidemment, je note que la Standard Edition intervertit parfois l'ordre des phrases et, plus fréquemment encore, qu'elle fusionne des paragraphes sans le mentionner, ou en crée de nouveaux).

Une autre remarque s'impose également : alors que la Standard Edition occupe $81 \mathrm{~cm}$ sur l'étagère d'une bibliothèque, les Gesammelte Werke, dont l'appareil éditorial est plus pauvre, prennent exactement la moitié de cet espace. Or c'est le contraire qui se produit avec les concordances. L'ouvrage allemand, qui a l'épaisseur d'un annuaire téléphonique métropolitain, couvre près de $46 \mathrm{~cm}$, alors que l'anglais est de $41 \mathrm{~cm}$. Ces mesures contradictoires s'expliquent toutefois à la lumière des particularités contrastantes des langues allemande et anglaise. L'allemand étant, de nature, particulièrement sujet aux inflexions de la langue - et parce qu'il recèle un potentiel extraordinaire pour la création de mots composés -, il y a beaucoup plus de variantes de mots clés en 
allemand qu'en anglais. Un exemple mineur, mais révélateur, est le mot anglais " ask ", que l'on retrouve sous deux autres formes, verbale et adjectivale, dans la Concordance : " asked " et " asking ". En contraste, douze variantes de l'allemand " fragen * apparaissent dans la Konkordanz : " frag ", " frage ", " fragend ", * fragende ", * fragenden ", "fragst ", « fragt ", " fragte ", " fragten ", " gefragt ", " gefragte * et " gefragten *. Aussi le nombre d'entrées lexicales dans la Konkordanz excède-t-il considérablement celui de sa cousine anglaise.

La différence entre les deux langues en question a évidemment laissé d'autres traces. La Concordance anglaise répertorie de manière pratique les 1840 mots qui apparaissent au moins cent fois dans l'index; dans la Konkordanz allemande, d'après mes calculs, seuls 1636 mots apparaissent aussi souvent. Là encore, la Concordance anglaise élimine 156 des mots les plus communs, parmi lesquels * again ", " am *, * each ", " ever ", " now " et * often ". Pourtant, les équivalents allemands de ces six mots ne font pas partie des 192 mots les plus fréquemment utilisés que les éditeurs de la Konkordanz ont choisi d'éliminer. Un exemple plus parlant tourne autour de l'absence de " $I$ " et \& it " dans la Concordance. Respectant la nature lexicale de leur langue, les éditeurs allemands ont laissé tomber " $i c h$ " et " es ", mais ont retenu leurs formes capitalisées :

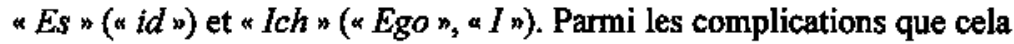
implique, il y a le fait que le pronom de la première personne du singulier, en allemand, doit être capitalisé en début de phrase. Illustrant en partie l'écart frappant qui peut naître de cette complication, * $I c h$ * apparaît avec la plus grande fréquence dans la Konkordanz, soit 6543 fois, alors que " ego " compte 2671 occurrences dans la Concordance. En contraste, "dream " est le mot qui revient le plus fréquemment dans la Concordance, apparaissant 6113 fois; dans la Konkordanz, "Traum " n'a que la septième plus haute fréquence d'apparition, soit 2826 occurrences. Or ce qui explique la disparité de cette * itémisation * lexicale, c'est la décision des éditeurs de la Concordance d'inclure des mots tels que $\alpha$ dream-thoughts " et $\alpha$ dream-content " sous la seule entrée de " dream ", alors que dans la Konkordanz chaque terme respectif ( Traumgedanken et "Trauminhalt ") profite d'une indexation individuelle, distincte de * Traum $*$. 
Les listes suivantes d'autres mots répertoriés avec des fréquences contrastantes peuvent également mettre en garde tout chercheur qui entretiendrait de trop grandes attentes en ce qui a trait aux correspondances :

$\begin{array}{ll}\text { Konkordanz } & \begin{array}{l}\text { Concordance } \\ \text { father (2 182) }\end{array} \\ \text { Vater (1 680) } & \text { repression (1 184) } \\ \text { Verdrängung (1 032) } & \text { hysteria (1 129) } \\ \text { Hysterie (919) } & \text { mother (1 447) } \\ \text { Mutter (1 357) } & \text { memory (1 142) } \\ \text { Erinnerung (864) } & \text { libido (1 038) } \\ \text { Libido (777) } & \text { God (620) } \\ \text { Gott (372) } & \text { phantasy (662) } \\ \text { Phantasie (516) } & \text { religion (510) } \\ \text { Religion (365) } & \text { death (839) } \\ \text { Tod (335) } & \text { Moses (330) } \\ \text { Moses (254) } & \text { affect (523) } \\ \text { Affekt (231) } & \text { cathexis (445) } \\ \text { Besetzung (228) } & \text { penis (368) } \\ \text { Penis (220) } & \text { superego (375) } \\ \text { Überich (220) } & \text { narcissism (185) } \\ \text { Narzissmus (185) } & \text { vagina (46) } \\ \text { Vagina (36); Scheide (9) } & \text { clitoris (48) } \\ \text { Klitoris (39) } & \end{array}$

On voit que les écarts entre les deux colonnes mettent en lumière quelques différences cruciales entre les lexicographies allemande et anglaise, de même qu'entre le Freud allemand et le Freud de la Standard Edition. L'entrée " father, Vater " illustre certaines des complications au niveau des comparaisons entre les colonnes. Il y a, dans la Concordance anglaise, six entrées distinctes concernant le mot "father ": "fatherland ", " fatherless ", « fatherly ", " father's ", * fathers * et * fathers' "; dans

\footnotetext{
${ }^{2}$ Huit fois sur neuf a Scheide * peut être un synonyme de a Vagina » et Strachey le traduit ainsi, à l'exception d'une oceurence où il le traduit de manière inexacte par « vulva * (11:315/16:305) - je ferai ainsi dorénavant référence au numéro du volume et à la page des Gesammelte Werke en premier lieu, puis à la Standard Edition. Strachey traduit correctement la seule autre occurrence de \& Scheide " par "dagger-sheath " $(19: 582 / 12: 185)$.
} 
l'ouvrage allemand, il y a près de cent entrées qui incluent le mot "Vater ", ce qu'explique la nature même de la langue allemande, ses inflexions affectant non seulement les diverses variantes de "Vater ", mais également la multitude de ses nombreux composés.

Tout en gardant à l'esprit les différences entre l'allemand et l'anglais, le lecteur pourra néanmoins noter les fréquences comparatives de " father ", " mother ", " penis ", " vagina " et " clitoris ", et sera frappé par l'énorme déséquilibre qui marque les fondements de l'exploration freudienne de la sexualité. On peut remarquer, de plus, la coïncidence pour le moins curieuse faisant que " penis " et "Überich " (" superego n) apparaissent exactement le même nombre de fois dans les Gesammelte Werke - hasard remarquable, et (mal?)heureux. $\hat{A}$ un autre niveau, un bel exemple de réflexion appropriée entre les langues apparaît avec les termes allemand et anglais pour narcissisme; non seulement sonnent-ils pareil, mais ils partagent la même fréquence d'apparition. Les analystes de diverses tendances pourraient poursuivre d'autres comparaisons révélatrices. Concordances en main, les Kleiniens pourraient vouloir comparer l'utilisation répétée que fait Freud de termes comme " restore * ( wiederherstellen $")$, " restitute " ( wiedergeben *) et de leurs variantes. Les Kohutiens, pour leur part, pourraient étudier l'usage fréquent que fait Freud du mot "empathy" ( $\alpha$ Einfuhlung ") dans ses formes verbales et substantivées.

Pour illustrer plus avant la nécessité de procéder avec précaution lorsqu'il est question de la fréquence des mots dans la Concordance, je vais concentrer mon attention sur deux termes centraux. Le premier provient d'une révélation autobiographique bien connue de Freud voulant que sa gouvernante ait été l'« Urheberin " de ses névroses, expression traduite par " primary originator " dans la Standard Edition $(1: 261)$. Étudions maintenant de plus près la liste complète des traductions dans la Standard Edition des diverses récurrences du masculin et du féminin "Urheber ", "Urheberin ", ainsi que de leurs substantifs dérivés :

Urheber : 1:486- those responsible * $(3: 254) ; 2: 38$ - * source * (4:6); $2: 149-\alpha$ are given $\times(4: 144) ; 5: 23$ - [non traduit] $(7: 265)$; $4: 172$ - chief actor * $(6: 156) ; 6: 21-*$ originated $*(8: 22) ; 6$ : 159 - originators * $(8: 142) ; 6: 160-$ * inventors " $(8: 143) ; 6: 162$ — * creators " $(8: 145) ; 8: 272-$ * first author * $(12: 38) ; 8: 272 \mathrm{n}-$ * first author * (12:38); $8: 280 \mathrm{n}-$ * first author * (12:45n); $10: 45$ 
— originator (14:8); $11: 42-\alpha$ the person responsible ( $(15: 49)$;

$11: 140-$ * originator... originators $*(15: 140) ; 12: 16-$ * instigators " (17:148); $13: 224$ - * originated by * $(18: 248) ; 14$ : $212-$ " murderer " $(20: 185) ; 15: 33-$ \& inventor * $(22: 32) ; 19: 110$ - *authors * $(1: 76) ; 19: 610-$ " creator * $(5: 361 \mathrm{n})$.

Urheberin : $4: 87$ - * woman responsible * $(6: 86) ; 5: 222$ - «prime author $\approx(7: 62)$.

Urhebern : $9: 191$ - * those who performed * $(13: 159)$.

Urheberrolle $: 8: 306-\approx$ leading part $*(12: 69)$.

Urhebershaft : $2: 50-\wedge$ responsibility $\times(4: 48)$.

Une conclusion incontestable s'impose à propos de $\alpha$ Urheber , un des termes les plus significatifs de l'autobiographie de Freud. Même si "Urheber " possède une certaine valeur en tant que point de référence dans l'œuvre de Freud, un lecteur qui ne dépendrait uniquement que de la Standard Edition et, a fortiori, de la Concordance, ne pourrait jamais le deviner.

Le deuxième terme qui mérite une plus grande attention illustre comment les sujets de la honte et de la culpabilité sont influencés selon qu'on les aborde en allemand ou en anglais. Le mot même de $\alpha$ Scham *, en allemand, signifie à la fois la honte et les parties génitales. Freud souligne d'ailleurs que, culturellement, le fait de donner aux parties génitales le nom d'un objet de honte se retrouve dans le latin " pudendax, littéralement : " les choses dont on doit avoir honte " (comparez 8 : $166 / 11: 96$ et $12: 236 / 17: 225)$. Freud cite également un auteur qui adopte cet usage (19:295/12:198). Mais si l'on tient compte, par contre, de l'exaspération répétée de Freud à l'égard du sentiment de honte dans les mœurs sexuelles viennoises, on peut se demander s'il utilise jamais de luimême le mot "Scham " dans son sens génital. On peut se rappeler que Freud a déjà dit avec défi : a Rien ne pourrait être plus éloigné de mes propres convictions ou des opinions théoriques que je défends en neuropathologie que de considérer la vie sexuelle comme quelque chose de honteux, dont ne devrait se préoccuper ni un physicien ni un chercheur 
scientifique ${ }^{3}$ (Freud, 1901, p. 606). Or le fait est que Freud fait preuve d'une cohérence évidente : lui-même n'utilise jamais directement le seul mot de "Scham " au sens de parties génitales.

À cette étape de notre examen, force est de constater que la Konkordanz s'avère indispensable s'il nous faut arriver à une autre réponse plus englobante. Freud n'utilise qu'en sept occasions le terme a Scham * dans un sens sexuel, et alors seulement quand il forme un nom composé. Ces occasions, parce qu'elles sont si rares, méritent toute notre attention : « Schamberg ", littéralement montagne de la honte - * Mons Veneris " (11 : 197/15 : 193); "Schamhaar „- " pubic hair »(2 : 90/4: 86); "Schamhaaren "- " pubic hairs" (5:262/7:99); "Schamlippen ", littéralement lèvres de la honte - « the labia" (2::392/5:387); « Schamspalte ", littéralement fente de la honte - * labia majora (7 : 179/9:217); "Schamteile ", littéralement parties honteuses - "the genitals" (13:62/ $18: 57)$; et "Schamgürtel ", littéralement ceinture de la honte - « athletic support-belt ( $14: 316 / 21: 156)$. Comme il s'agit pour nous de cerner des révélations exceptionnelles en ce qui a trait à la psyché de Freud, nous pouvons d'emblée éliminer quatre des sept dernières occurrences de "Scham " dans un mot composé, soit parce que Freud cite alors quelqu'un d'autre ( Schamhaar ", « Schamteile $e^{4}$ ), soit parce qu'il explique un usage symbolique ou linguistique approprié à des rêves particuliers ("Schamberg ", "Schamlippen ").

Je voudrais suggérer que les trois autres occurrences d'un mot composé avec « Scham " sont surdéterminées. On peut se demander, en premier lieu, pourquoi Freud n'utilise pas un synonyme pour "Schamhaar" dans le cas Dora comme il le fait en d'autres occasions :

${ }^{3}$ Ma traduction (N.d.T.).

${ }^{4}$ La traduction allemande du Symposium de Platon dont Freud se sert dans Audelà du principe de plaisir peut se lire comme suit : " Tout de ces premiers hommes était double : ils avaient... deux parties privées [* Schamieile "] ". Bien que je n'aie pas vérifié a mêne le texte grec, je pourrais ajouter que la forme plurielle de aidolon, le mot grec pour honte, signifie parties génitales. 
" crines pubis " $\left(3: 371,382 ; \mathrm{n} / 5: 366 \mathrm{n}, 377^{5}\right)$, «enitalhaare " (19: 564/10:311) et * Genitalbehaarung * (11:157/15:156; $14: 314 / 21$ : $155 ; 15: 142 / 22: 132 ; 19: 567 / 10: 315)$ ? Ensuite, pourquoi n'utilise-t-il pas, comme il le fait ailleurs, un synonyme pour "Schamspalte * dans "Les theories sexuelles infantiles "? Même si elles sont particulièrement exceptionnelles, ces deux demières désignations de la honte, utilisées pour décrire des parties du corps féminin, deviennent significatives quand, d'une part, on considère que Freud ne s'est jamais servi d'une telle désignation pour décrire les parties du corps masculin (comme "Schamglied", littéralement le membre de la honte, pour pénis) et, d'autre part quand on garde à l'esprit que l'on retrouve beaucoup plus fréquemment le mot pénis dans les Gesammelte Werke que ses contreparties féminines. Mais il est vrai que le mot composé qu'il nous reste à examiner concerne l'homme; de manière assez pertinente, par contre, le mot en question - * Schamgürtel $"$, ceinture de support athlétique - s'avère être l'appareillage d'un fétichiste masculin!

Pendant des années les éléments lexicaux considérés dans le dernier paragraphe sont demeurés cachés parmi le million de mots et plus des Gesammelte Werke. Or grâce à l'informatique, j'ai été en mesure de suivre à la trace la cohérence spectaculaire de Freud et, de plus, de relever un minimum de dérives - presque rien - dans deux poignées d'éléments lexicaux. C'est ainsi que nous avons pu observer l'inconscient de Freud, non pas dans une quelconque éruption dramatique, mais dans une "Regung" ", c'est-à-dire dans l'origine d'un mouvement, un remuement (pour des mouvements plus intenses comme l'excitation ou l'agitation, on utiliserait les mots "Erregung " et "Aufregung "). En traduisant * Regung " par * impulse ", terme beaucoup plus défini et intense, Strachey a empêché les lecteurs de langue anglaise de reconnaître la place centrale et les implications majeures de ce mot dans le vocabulaire de Freud (nos revues sont submergées par des études cliniques concernant les impulsions, mais aucune ne s'attarde à ce remuement). En examinant les

${ }^{5}$ Je devrais préciser que la première occurrence de « crines pubis * $(2: 371)$ que je cite a échappé au traitement informatique et qu'il n'est donc pas relevé par les éditeurs dans leur Konkordanz.

"Voir Darius Omston (1982), de même que les entrées * Regung *, * impulse * et " stirring * dans son index (1992). 
quelque 400 occurrences et plus de * Regung ", au singulier comme au pluriel, nous pouvons non seulement en apprendre plus sur la manière dont Freud percevait l'inconscient, mais également sur son écoute en clinique et, par le fait même, sur la nôtre...

Il serait peut-être temps, en ce sens, d'entreprendre une réflexion globale sur la montée d'un désordre lexicographique en psychanalyse. De manière plus positive, nous sommes maintenant dans une meilleure position pour entreprendre une psychanalyse de la traduction de Strachey, analyse qui, en retour, serait une des bases possibles pour entreprendre une étude des diverses tendances en psychanalyse à travers les frontières nationales et linguistiques. La fortune que connaît l'interprétation de l'œuvre de Freud, en allemand comme en d'autres langues, en fait une histoire fascinante parsemée de considérations scientifiques, politiques, sociales et, oui, économiques. Après tout, il est extrêmement significatif que la Standard Edition, terminée au milieu des années soixante, ait fortifié une emprise anglo-américaine dans la profession de la psychanalyse à une époque où ce même axe, justement, dominait le monde politique et économique. Parallèlement aux retards théorique et technique qui assiègent le développement de notre profession, voilà maintenant que la menace aussi un retard d'ordre lexicographique. Une fixation linguistique lourdement investie en divers quartiers est remise en question alors même que nous approchons du prochain siècle. Avec la destitution en cours de l'hégémonie du dollar américain sur les marchés internationaux, assistons-nous également à la destitution (douloureuse, patriotisme oblige) de l'anglais comme incontestable lingua franca et marque de commerce de la psychanalyse? Un libre marché serait-il en train de se dessiner?

Je voudrais conclure sur une autre note à propos du futur, et à cette fin j'emprunterai une citation de mon dernier livre (1996) qui contient quantité de nouveaux commentaires sur le texte allemand du cas Dora. On y retrouve un exemple spectaculaire des subtils artifices phoniques de Freud dans son explication du principe de contiguité (par lequel un $a$ et un $b$ équivalent à $a b$ ). Jai remarqué que, dans le texte allemand de son explication, des mots tels que "Abwechslung ", "Abhängigkeit ", "Abwesenheit ", " Abneigung ", en vertu de leur préfixe identique, deviennent plus ou moins synonymes l'un de l'autre. Mes ajouts 
entre crochets à la traduction de Strachey mettent en lumière les aspects associatifs cachés du discours de Freud:

\begin{abstract}
Dora suddenly brought in an allusion to her own alternations [Abwechslung] between good and bad health during the first years of her girlhood at B-; and I was thus driven to suspect that her states of health were to be regarded as depending [Abhăngigkeit] upon something else, in the same way as Frau K's. (It is a rule of psychoanalytic technique that an internal connection which is still undisclosed will announce its presence by means of a contiguity $-a$ temporal proximity - of associations; just as in writing, if " $a$ " and $* b$ " are put side by side, it means that the syllable $a b b$ is to be formed out of them). Dora had had a very large number of attacks of coughing accompanied by loss of voice. Could it be that the presence or absence [Abwesenheit] of the man she loved had had an influence upon the appearance and disappearance of the symptoms of her illness? ... Her illness was therefore a demonstration of her love for $K$, just as his wife's was a demonstration of her dislike [Abneigung, 198/39]).
\end{abstract}

L'étude de l'inconscient et du préconscient de Freud en est encore dans son enfance. Javancerais en ce sens que ses schèmes syntaxiques, voire davantage encore ses schèmes phoniques, seront la cible d'études informatiques révolutionnaires dans le prochain siècle. C'est un monde merveilleux qui nous interpelle ainsi, celui d'un enfant encore jeune, l'ordinateur, grâce auquel nous pourrons étendre nos perspectives et élargir nos horizons. Sans doute nous faudra-t-il de nouveau apprendre à voir, et de nouveau apprendre notre abc.

Université de Montréal

\title{
Références
}

FREUD, Sigmund (1888). Preface to the translation of Bernheim's Suggestion. Standard Edition I (Gesammelte Werke 19).

-(1893-95). Studies on Hysteria. S.E. 2 (G.W. 1).

- (1900). The Interpretation of Dreams. S.E. 4-5 (G.W. 2-3). 
- (1901). The Psychopathology of Everyday Life. S.E. 6 (G.W. 4).

- (1905a). Fragment of an Analysis of a Case of Hysteria. S.E. 7 (G.W. $5)$.

- (1905b). Jokes and their Relation to the Unconscious. S.E. 8 (G.W. 6).

$-(1906)$. My Views on the Part Played by Sexuality in the Atiology of the Neuroses. S.E. 7(G.W. 5).

$-(1908)$. On the Sexual Theories of Children. S.E. $9($ G.W. 7).

$-(1909)$. Notes upon a Case of Obsessional Neurosis (Addendum). S.E. 10 (G.W. 19).

- (1910). Leonardo da Vinci and a Memory of his Childhood. S.E. 11 (G.W. 8).

- (1911). Psychoanalytic Notes on an Autobiographical Account of a Case of Paranoia. S.E. 12 (G.W. 8).

-(1913). Totem and Taboo. S.E. 13 (G.W. 9).

- (1914). On the History of the Psychoanalytic Movement. S.E. 14 (G.W. 10).

- (1915-17). Introductory Lectures on Psychoanalysis. S.E. 15-16 (G.W. 11).

- (1917). A Childhood Recollection from Dichtung und Wahrheit. S.E. 17 (G.W. 12).

- (1919). The "Uncanny ». S.E. 17 (G.W. 12).

-(1920). Beyond the Pleasure Principle. S.E. 18 (G.W. 13).

- (1922). Some Neurotic Mechanisms in Jealousy, Paranoia and Homosexuality. S.E. 19 (G.W. 13). 
-(1926). The Question of Lay Analysis. S.E. 20 (G.W. 14).

-(1927). Fetishism. S.E. 21 (G.W. 14).

-(1933). New Introductory Lectures on Psycho-Analysis. S.E. 22 (G.W. 15).

- (1950). Extracts from the Fliess Papers. S.E. 1.

- (1957). Dreams in Folklore. S.E. 12 (G.W. 19).

MAHONY, Patrick (1986). Freud and the Rat Man. New Haven, CT, Yale University Press.

-(1987a). Freud as a Writer, second enlarged edition. New Haven, CT, Yale University Press.

- (1987b). Psychoanalysis and Discourse. London, Tavistock and Methuen.

- (1989). On Defining Freud's Discourse. New Haven, CT, Yale University Press.

- (1995). Les hurlements de l'Homme aux loups, deuxième édition révisée. Paris, Presses Universitaires de France.

- (1996). Freud's Dora: A Psychoanalytic, Historical, and Textual Study. New Haven, CT, Yale University Press.

ORNSTON, Darius (1982). « Strachey's Influence : A Preliminary Report n. International Journal of Psychoanalysis, 63 : 409-426.

- (edit.) (1992). Translating Freud. New Haven, CT, Yale University Press.

RÉSUMÉ : Discordance dans la concordance : ressacs de la traduction - L'auteur décrit d'abord le dessein et les propriétés de la Konkordanz, monumental ouvrage de référence paru en 1996. Véritable 
événement éditorial dans le champ psychanalytique, cet ouvrage donne accès aux termes clés des Gesammelte Werke de Freud. L'auteur compare ensuite cette Konkordanz allemande et la Concordance anglaise, et relève des discordances révélatrices sur le plan lexicographique. Cette comparaison confirme la remarquable cohérence de la conceptualité freudienne, d'une part, et la nécessité d'entreprendre une a psychanalyse de la traduction de Strachey " d'autre part, car les processus de l'inconscient et du préconscient ne passent pas de la même manière d'une langue à l'autre. L'anglais doit-il rester la lingua franca de la psychanalyse?

\begin{abstract}
Discordance Between the Concordances : Translation on the Rebound - The author first describes the design and characteristics of the Konkordanz, a monumental work of reference published in 1996. Constituting nothing less than a majestic editorial event in the field of psychoanalysis, this work is useful as a location guide and frequency register of the key terms in the Gesammelte Werke of Freud. The author then compares this German Konkordanz and the English Concordance, and proceeds to highlight a certain number of revelatory discordances on the lexicographical level. This comparison confirms the remarkable coherence of Freud's conceptualization on one hand, and on the other hand underlines the necessity of undertaking "a psychoanalytic translation of Strachey," for the processes of the unconscious and preconscious are variously deflected in the passage from one language to another. Whence the urgent question : should English remain the lingua franca of psychoanalysis?
\end{abstract}

Patrick J. Mahony : 1297, St-Viateur, Outremont (Québec) H2V $1 Z 2$. 